\title{
DVPW
}

\section{Laudatio: Verleihung des DVPW-Förderpreises 2011 für Dissertationen an Thorsten Faas}

Auch 2011 standen für die Auswahl des DVPW-Förderpreises für Dissertationen viele herausragende Arbeiten zur Verfügung. Insgesamt wurden 18 Studien nominiert. Mit Hilfe der jeweils von einschlägigen Experten eingeholten externen Fachgutachten hat sich die Jury entschieden, den Preis an Thorsten Faas für seine Dissertation „Arbeitslosigkeit und Wahlverhalten. Direkte und indirekte Wirkungen auf Wahlbeteiligung und Parteipräferenzen in Ost- und Westdeutschland“ zur vergeben. Die Arbeit wurde 2008 an der Universität Duisburg-Essen auf Grundlage der dortigen Gutachten von Rüdiger Schmitt-Beck und Stefan Liebig als Promotionsschrift angenommen. Sie ist 2010 als Band 17 der renommierten Reihe „Studien zur Wahl- und Einstellungsforschung“ (Nomos, Baden-Baden) erschienen.

Die Arbeit greift ein Thema auf, von dem man angesichts der augenscheinlichen politikwissenschaftlichen Relevanz vermuten könnte, dass bereits erschöpfende Erkenntnisse dazu vorliegen: Faas problematisiert das Verhältnis zwischen Arbeitslosigkeitserfahrungen und Wahlverhalten in Deutschland. Ein Ausgangspunkt ist die Beobachtung der gleichzeitigen Unterschiede der Arbeitsmärkte in Ost- und Westdeutschland und der Wahl- und Parteiensysteme in den alten und den neuen Bundesländern. Gerade vor dem Hintergrund der kontrovers diskutierten Thesen zu Zusammenhängen zwischen Arbeitslosigkeit und Stimmenanteilen der NSDAP in der Weimarer Republik liegt es nahe, die möglichen Beziehungen empirisch zu überprüfen. Dennoch spielte bis zu der hier ausgezeichneten Arbeit die Frage nach politischen Folgen der Arbeitslosigkeit in den letzten 20 Jahren in der deutschen Politikwissenschaft nur eine geringe Rolle. Die Dissertation behandelt somit ein Problemfeld, das von großer fachlicher und politischer Bedeutung ist. Sie verspricht gleichzeitig, eine wesentliche Forschungslücke zu schließen.

Faas knüpft bei der Strukturierung seiner Untersuchung an die Ergebnisse der meist schon älteren Studien und Theorien an, die vor allem nahelegen, neben direkten auch kontextbasierte Arbeitslosigkeitserfahrungen zu betrachten. Aus der Literatur leitet die Arbeit ein komplexes Kausalmodell ab. Danach wirken unmittelbare und kontextbasierte Erfahrungen vermittelt über Einkommen und die Wahrnehmung der Wirtschaftlage auf Gerechtigkeitsempfindungen und allgemeine Lebenszufriedenheit. Diese vermittelnden Faktoren beeinflussen wiederum Einstellungen zur eigenen Rolle und zur Politik, was letztlich auf Wahlbeteiligung und Parteiwahl wirkt.

Zur empirischen Umsetzung nutzt Faas Umfragedaten aus den Jahren 1977 bis 2006, die seit der Wiedervereinigung für Ost- undWestdeutschland getrennt aus- 
gewertet werden. Viele der Ergebnisse überraschen zunächst nicht: Arbeitslosigkeit wirkt sich tatsächlich in der Regel negativ auf die Wahlbeteiligung aus. Spannend sind aber die Ergebnisse im Detail: So haben in der Sondersituation 1998 Arbeitslose ihre Unzufriedenheit ausnahmsweise nicht systematisch in Wahlenthaltung übersetzt. Unter den speziellen Rahmenbedingungen einer lange regierenden bürgerlichen Regierung konnte nicht die „Partei der Nichtwähler“ sondern die SPD profitieren. Interessant ist auch, dass dieser Mobilisierungseffekt der SPD nach den offenbar für viele Arbeitslose enttäuschenden Ergebnissen der rot-grünen Regierung schnell wieder verschwunden ist.

Auch die Ergebnisse für die Parteiwahl entsprechen zunächst den Erwartungen: In Zeiten bürgerlicher Regierungen tragen Arbeitslosigkeitserfahrungen dazu bei, sich linken Oppositionsparteien zuzuwenden. Entsprechendes gilt weitgehend auch für Zeiten sozialdemokratisch geführter Regierungen: Hier profitiert aber nicht die bürgerliche Opposition, sondern die als linker empfundene Opposition (in den 1980er-Jahren in Westdeutschland die Grünen, heute die Linkspartei) von der schlechteren Wahrnehmung wirtschaftlicher Lagen infolge von Arbeitslosigkeitserfahrungen. Auch die Parteipräferenzen werden jeweils von Kontextvariablen beeinflusst. Interessant ist aber, dass die Linkspartei (trotz ihrer unterschiedlichen Historie, Policies und Verankerungen) in Ost- und Westdeutschland gleichermaßen für unzufriedene Arbeitslose besonders attraktiv ist.

Die hier ausgezeichnete Dissertation geht in ihrem komplexen Anspruch und ihrer Methodik neue und überzeugende Wege. Die Studie erschöpft sich nicht in der Auswertung von Zusammenhängen auf der Mikroebene, sondern nutzt eine Vielzahl unterschiedlicher Daten zur systematischen Umsetzung ihres differenzierten Modells. Das Ergebnis ist zweifellos ein wesentlicher Schritt für die empirische Wahlforschung. Die Arbeit ist theoretisch und methodisch außerordentlich anspruchsvoll und kommt zu klaren und sprachlich und graphisch exzellent aufbereiteten Ergebnissen.

Thorsten Faas zeichnet sich über die Qualität seiner Dissertation hinaus als international orientierter, aktiver und erfolgreicher Wissenschaftler aus. Er hat auch über das Themenfeld der Arbeit hinaus bereits eine bemerkenswerte Zahl sehr gut platzierter Fachartikel veröffentlicht, die inzwischen breite Aufmerksamkeit gefunden haben. Die Mitglieder der Jury gratulieren dem Preisträger herzlich und wünschen ihm für die Zukunft weiterhin viel Erfolg. Wir bedanken uns außerdem bei den Kolleginnen und Kollegen, durch deren Fachgutachten die Auswahl ermöglicht wurde.

Maria Behrens (Wuppertal)

Nils C. Bandelow (Braunschweig)

Marcus Llanque (Augsburg) 